\title{
$20 \mathrm{MHz}$ Forward-imaging Single-element Beam Steering with an Internal Rotating Variable-Angle Reflecting Surface: Wire phantom and Ex vivo pilot study
}

\author{
David T. Raphael ${ }^{\varphi},{\text { Xiang } \mathrm{Li}^{\Phi}}$, Jinhyoung Park ${ }^{\Phi}$, Ruimin Chen ${ }^{\Phi}$, Hamid Chabok ${ }^{\Phi}$, Arthur \\ Barukh $^{\omega}$, Qifa Zhou ${ }^{\Phi}$, Mahmoud Elgazery ${ }^{\sigma}$, and K. Kirk Shung ${ }^{\Phi}$ \\ ${ }^{\varphi}$ Dept. of Anesthesiology, Keck School of Medicine, University of Southern California; President, \\ Sonoscrew, Inc., Valley Village, CA \\ $\Phi \mathrm{NIH}$ High-Frequency Ultrasound Transducer Resource Center, Dept. of Biomedical Engineering, \\ University of Southern California, Los Angeles, CA \\ $\sigma$ National Institute of Standards, Giza, Egypt \\ ${ }^{\omega}$ Calasers, Inc., Simi Valley, CA
}

\begin{abstract}
Feasibility is demonstrated for a forward-imaging beam steering system involving a singleelement $20 \mathrm{MHz}$ angled-face acoustic transducer combined with an internal rotating variableangle reflecting surface (VARS). Rotation of the VARS structure, for a fixed position of the transducer, generates a 2-D angular sector scan. If these VARS revolutions were to be accompanied by successive rotations of the single-element transducer, 3-D imaging would be achieved.

In the design of this device, a single-element $20 \mathrm{MHz}$ PMN-PT press-focused angled-face transducer is focused on the circle of midpoints of a micro-machined VARS within the distal end of an endoscope. The 2-D imaging system was tested in water bath experiments with phantom wire structures at a depth of $10 \mathrm{~mm}$, and exhibited an axial resolution of $66 \mu \mathrm{m}$ and a lateral resolution of $520 \mu \mathrm{m}$. Chirp coded excitation was used to enhance the signal-to-noise ratio, and to increase the depth of penetration. Images of an ex vivo cow eye were obtained. This VARS-based approach offers a novel forward-looking beam-steering method, which could be useful in intracavity imaging.
\end{abstract}

\section{Introduction}

B-mode ultrasonic single-element imaging systems rely upon the oscillatory motion of an acoustic transducer. The transducer motion can be achieved either by mechanically pivoted oscillatory steering, or through an electromagnetic linkage, with or without a reflecting surface (mirror). This oscillatory or rotational motion results in either a forward or sideviewing capability. Single-element sector probes are limited by poor near-field imaging because of reverberations between the transducer and the housing, and by a fixed focus [1].

\footnotetext{
(C) 2012 Elsevier B.V. All rights reserved.

Corresponding Author: David T. Raphael Telephone: 818-752-8643, raphael.david92@ gmail.com.

Publisher's Disclaimer: This is a PDF file of an unedited manuscript that has been accepted for publication. As a service to our customers we are providing this early version of the manuscript. The manuscript will undergo copyediting, typesetting, and review of the resulting proof before it is published in its final citable form. Please note that during the production process errors may be discovered which could affect the content, and all legal disclaimers that apply to the journal pertain.
} 
Alternatively, B-mode imaging can be achieved without motion through the use of a multiple-element array transducer. However, multiple-element transducers have a greater complexity of electrical interconnections [2].

In clinical interventional procedures, the operator inserts a needle-shaped device toward a tissue structure, and may make use of imaging guidance provided either by a B-mode ultrasound probe placed on the skin surface, or by MRI/CAT scans, and fluoroscopy. Ultrasound imaging of a deep target with such a surface probe can present significant problems at depths greater than $10 \mathrm{~cm}$, e.g., impaired resolution, tissue differentiation difficulties, and bone obstruction of view [3].

In ultrasound biomicroscopy (UBM) systems in the 20-100 MHz range, high tissue resolution can be obtained. However, the use of high frequencies results in limited penetration depth, and consequently UBM applications have been largely limited to dermatology and ophthalmology [4-6]. A possible solution to this depth problem is to place a high-frequency transducer on a needle tip and to advance it steadily through tissue, thus allowing continuous close-up imaging with undiminished spatial resolution at successively greater depths. Such a capability would allow the imaged anticipation of critical anatomical structures at a workable distance, and thereby prevent traumatic injury to these structures.

A motionless single-element transducer emits a single forward-looking ultrasonic A-mode beam that, without a steering mechanism, is inadequate for most clinical applications. Sideviewing intravascular ultrasound (IVUS) commercial devices are available that rotate a single element transducer to achieve circumferential imaging. A thin capacitative micromachined ultrasonic transducer (CMUT) has been reported which employs an annular-array intravascular ultrasound system (IVUS) for forward imaging [7]. Other CMUT arrays with front-end electronics have been miniaturized to fit inside channels as narrow as 5 -mm both within endoscopes and within flexible catheters [8]. As with this recent research, we sought to develop a B-mode forward-viewing single-element needle-shaped endoscope with a total diameter of $5 \mathrm{~mm}$.

Toward this end, we (1) develop a novel method of beam steering based upon a built-in rotating variable-angle reflecting surface (VARS) for use in 2-D and 3-D imaging, (2) fabricate a VARS prototype 2-D 'straight-ahead imaging' $20 \mathrm{MHz}$ needle device $5 \mathrm{~mm}$ in diameter, (3) conduct experimental water bath experiments with phantom wire structures with and without chirp-coded excitation, and (4) implement chirp-coded excitation for the imaging of an ex vivo cow eye.

\subsection{Angled-face transducers and the 'blind spot' problem}

The fabrication of angled-face single-element transducers has been reported [9-10]. A rotating angled-face transducer with face tilt angle $a$ can achieve partial forward-imaging in a 2-D plane, but cannot achieve 'straight-ahead' viewing, because it is limited by the presence of an acoustic 'blind' region (see Fig. 1a). After a complete transducer rotation, a 3 -D cone-shaped blind region is created in the forward direction (see Fig. 1b).

\subsection{The internal variable angle reflecting surface (VARS)}

We embarked on an effort to develop a method that would eliminate these conical blind regions associated with angled-face transducers, and that would allow 'straight-ahead' forward-imaging 2-D sector scanning. In this paper we present an approach that is based upon an internal variable-angle reflecting surface (VARS). To our knowledge, no research has been done on this type of unconventional type of reflecting surface. 
Such a pre-formed variable-angle reflecting surface (VARS) surface is capable of generating the desired angular variation that occurs with the sinusoidal sweep of a rotating mirror. It is sufficient merely to rotate the VARS structure at constant speed for a fixed position of the transducer, and a 2-D angular sector scan is generated. If these VARS revolutions are performed along with successive rotations of the single-element transducer itself, it is possible to achieve 3-D imaging. The single-element transducer can be rotated, and gearinterlinked, relative to the VARS structure. These devices should be amenable to further miniaturization.

\subsection{Internal VARS design and function}

For the sake of illustration, we consider in Fig. 2 the turret structure contained within the distal end of a cylindrical endoscope (outlined by the two solid black lines).

In Figure 2, the central axis of the transducer coincides with the central axis of the turret. The angled-face transducer emits a beam that, en route to the target and back, interacts with the interior surface of the uppermost wedge of the interposed turret. The turret is made up of multiple wedges with interior reflecting surfaces, each of which may be differently angled. Rotation of the turret and of the transducer arm are achieved with separate yet synchronized motorized mechanisms.

\subsection{Relation of the transducer face angle to the inner surface tilt angle}

The angled-face transducer has tilt angle $\alpha$. If the tilt angle of the uppermost undersurface is angle $\beta$, then the angle of the reflected beam RB that exits from the distal end of the endoscope toward the target is $\mathrm{RB}=\pi / 2-\alpha-2 \beta$. If we set $\alpha=45^{\circ}$, then $\mathrm{RB}=45^{\circ}-2 \beta$.

In Figure 3, let us assume a vector coordinate system whose origin is at the midpoint of the transducer face. The midpoint of the transducer face is the point where the dotted red line turns into a solid red line. In the $x-y$ plane of the diagram, we set the vector origin at this midpoint, i.e., transducer midpoint $=0 \mathbf{i}+0 \mathbf{j}$ (positive $\mathbf{i}$ points to the left, positive $\mathbf{j}$ is upwards). The distance $\mathrm{R}$ from the transducer face midpoint to the midpoint of the inner reflecting surface is given by

$$
R=\left(\begin{array}{ll}
R & \sin \alpha
\end{array}\right) i+\left(\begin{array}{ll}
R & \cos \alpha
\end{array}\right) j
$$

For the variable-angle reflecting surface of tilt angle $\beta$ and of length $b$, the outer edge ( $V$ outer) is the edge midpoint closest to the distal end of the endoscope. The inner edge (V inner) is the edge midpoint farthest away from the distal endoscope end. The midpoint coordinates of these two edges are:

$$
\begin{gathered}
V \quad \text { outer }=\left(\begin{array}{lllll}
R & \sin \alpha+b / 2 & \cos \beta
\end{array}\right) i+\left(\begin{array}{llll}
R & \cos \alpha+b / 2 & \sin \beta
\end{array}\right) j \\
V \quad \text { inner }=\left(\begin{array}{lllll}
R & \sin \alpha-b / 2 & \cos \beta
\end{array}\right) i+\left(\begin{array}{llll}
R & \cos \alpha-b / 2 & \sin \beta
\end{array}\right) j
\end{gathered}
$$

The vector difference between the outer and inner VARS boundaries is given by

$$
V \quad \text { outer }-V \quad \text { inner }=\left(\begin{array}{lll}
b & \cos \beta
\end{array}\right) i+\left(\begin{array}{ll}
b & \sin \beta
\end{array}\right) j .
$$

The implications of Eqs. 2-4 are that the VARS strip will rotate, and depending on the point of view of an observer, may appear to become thicker or thinner when viewed from different directions. 


\subsection{2-D imaging with stationary angled-face transducer and rotating VARS}

In Figure 4, the beam of the inner transducer is always directed toward the undersurface of the wedge in the uppermost position. The transducer is behind the turret as shown earlier in Figure 2. As the turret rotates, the uppermost wedge undersurfaces are rotated sequentially into position to receive the beam from the stationary angled-face transducer. Starting with mirror zero, the transducer then rotates so that successive mirrors are placed directly below the transducer-first, mirror 0 , then mirror 1 , then mirror 2, etc. so that different reflection angles are obtained.

Assume undersurface $\mathrm{M} 0$ has a $\beta=0^{\circ}$ angle tilt relative to the horizontal such that $\mathrm{RB}=$ $45^{\circ}$. The beam emitted from the distal transducer is reflected in a forward $45^{\circ}$ direction below the horizontal. The turret is rotated so that now mirror 1 is in the uppermost position. Assume surface M1 has a $2.5^{\circ}$ angle tilt to the horizontal. The redirected forward beam is now at a $40^{\circ}$ angle from the horizontal. The turret is rotated so that mirror 2 is now advanced into the zero position. Assume mirror 2 has a $5^{\circ}$ angle tilt to the horizontal. The reflected forward beam is now at a $35^{\circ}$ angle from the horizontal. And so forth and so on. The wedge undersurface reflection point and the points $\mathrm{A}, \mathrm{B}$, and $\mathrm{C}$ serve to outline the 2-D angular sector scan image.

If the beam of the angled-face transducer is directed upwards, the reflected beams from the wedge undersurfaces will provide an image of the lower field. Upon a $180^{\circ}$ rotation of the angled-face transducer, the image of the upper field is generated. The VARS goes through a complete revolution to create a $2-\mathrm{D}$ sector image.

\subsection{3-D Imaging with Rotating Angled-Face Transducer and Rotating VARS}

For a fixed position of the turret, let us now rotate the transducer by angle $\varphi$ about the central axis (see Figure 5). The effect of a transducer at angle $\varphi$, after successive turret rotations, is to create a new series of circular arc points-D, E, F-at an opposite angle $\varphi$ relative to the vertical axis. This creates another 2-D angular scan.

For the transducer rotated by $2 \varphi$, a new series of arc points- $\mathrm{G}, \mathrm{H}, \mathrm{I}$-are generated at an angle $2 \varphi$ with respect to the starting orientation (see Figure 6). This process is continued for increments of angle $\varphi$ until the desired scanning sector is covered. In the horizontal plane, mirror M0 traces out an arc that includes points A, D, and G. Mirror M1 traces out an arc that includes points B, E, and H. Mirror M2 traces out an arc of points C, F, and I. For N successive $\varphi$ rotations of the transducer, each of which is followed by a $360^{\circ}$ rotation of the turret, one obtains $\mathrm{N} \times \mathrm{M}$ image lines, where $\mathrm{N}=$ number of transducer positions, and $\mathrm{M}=$ number of turret mirrors.

\subsection{The VARS reflecting surface: discretized and smooth}

In the discussion above, each discrete wedge contributes a discrete reflecting surface to the overall internal reflecting surface of the turret. Each wedge's reflecting surface exhibits a fixed-torsion angle offset relative to the immediately adjacent wedge's reflecting surface. For a fixed-position transducer, rotation of the turret causes a successive discrete increase in the tilt (torsion) of successive mirrors. The net effect is to cause each successive incident beam to be reflected from the midpoint of each successive wedge, thus producing successive different reflection angles. These multiple A-mode beams can be used to generate a successive series of points that create an angular sector scan in a forward imaging direction. The focal point of the maximal echo in the imaging plane is unaffected by the interposition of a reflecting mirror. The VARS reflecting surface is comparable to that of a constrained helix. The VARS can exhibit the full angular variation of a helix, but the midpoints of all successive reflecting regions are constrained to a circle on the perimeter of a circular disc, as 
is evident from Figure 7. All VARS reflecting surface midpoints together are found on a circle (the dark red line in Fig. 7). For the purpose of illustration, in Fig. 7, the VARS surface is discretized into 21 reflecting surfaces. For any specific VARS midpoint on the circle, there is a specific torsion angle associated with it. The circle of VARS midpoints provides the axes of rotation for any and all possible reflecting surfaces.

Rotation of the VARS structure can generate successive increases in torsion angle that occur as the quasi-helix ascends from its zero torsion angle starting point to its maximal torsion angle $\beta_{\max }$. In reverse manner, the torsion angle can decrease back to zero, and descend further to a minimum of $\left(-\beta_{\max }\right)$, and finally back again to the zero starting point. Hence the VARS can generate a torsion angle variation of one-half ascending helix and one-half descending helix. That is, unlike a helix where the trajectory never returns to its starting position, the VARS quasi-helix can return to its original starting position and, in that sense, is constrained.

In a beam sweep, the VARS can be designed to have an angular variation that is purely ascending (increasing torsion or tilt angle) or purely descending (decreasing torsion), which is subject to reversibility of direction upon a counter-rotation. Hence a conventional 2-D beam sweep can feature a monotonically increasing or monotonically decreasing torsion angle. Lastly, we note that the design of the VARS can be specified to allow regions of arbitrarily increasing or decreasing VARS torsion angles. This arbitrary design option is non-standard and merits further exploration.

In summary, we make these observations:

1. If the transducer is stationary, the rotating VARS generates a single 2-D angular sector scan, which is equivalent to that produced by a linear array scanner.

2. If the transducer is likewise rotated, the rotating VARS generates multiple 2-D sectors that can achieve a 3-D volume reconstruction.

All the above observations concerning the properties of VARS surfaces were gained with the use of a discretized theoretical model. We now consider the laboratory fabrication of a smooth-surfaced VARS surface and its incorporation into a needle endoscope.

\section{Materials and methods}

Selection of a piezoelectric material for the transducer with the best possible performance characteristics is critical. The $20 \mathrm{MHz}$ transducer was formed from crystals of $\mathrm{Pb}\left(\mathrm{Mg}_{1 / 3} \mathrm{Nb}_{2 / 3}\right) \mathrm{O}_{3}-\mathrm{PbTiO}_{3}(\mathrm{PMN}-\mathrm{PT})$, a piezoelectric relaxor-PT material, which has an excellent longitudinal coupling coefficient $\left(\mathrm{k}_{33}\right)>90 \%$, a dielectric constant ranging from 1000 to 5000 with low dielectric loss $<1 \%$, and an exceptional piezoelectric coefficient $\mathrm{d}_{33}>2000 \mathrm{pC} / \mathrm{N}$ [9-10].

The reflection from the VARS surface requires that the beam be focused exactly at the midpoint of the VARS surface. For the focused $20 \mathrm{MHz}$ transducer, the focus was at 7.5 $\mathrm{mm}$, with an approximate working distance of 1.0 to $1.5 \mathrm{~cm}$ in the water bath. From a procedural standpoint, this is a distance that might be suitable when imaging through soft tissue, e.g., in the guided advancement of a needle.

\subsection{Design and micro-machining of a smooth VARS surface}

The design for a smooth VARS inner surface geometry for the endoscope was specified and created with use of a CAD-CAM system. The smooth VARS surface would exhibit a monotonically increasing tilt angle range of $30^{\circ}$ (from $-15^{\circ}$ to $+15^{\circ}$ ), thus allowing an imaging sector span of $60^{\circ}$. 
Micro-machining and shaping of the VARS surface were accomplished with wire electric discharge machining (EDM) with the use of a five-axis Charmilles Robofill 240 device at the facility of California Lasers Inc., Simi Valley, CA. This involved identifying the proposed circle of VARS midpoints, establishing a vertical line through the midpoints, and making the EDM contour cut that produced the desired reflecting angles. In terms of smoothing the VARS surface, a two-stage abrasives polishing centrifugal disk deburring machine with ceramic media was used to achieve an initial smooth polish $(<3 \mu \mathrm{m})$, followed by a surface finish $(<1 \mu \mathrm{m})$.

\subsection{Focused angle-faced transducer}

The PMN-PT transducer (see Fig. 8) is of aperture size $1.15 \mathrm{~mm} \times 1.63 \mathrm{~mm}$, and has a 7.5 $\mathrm{mm}$ focus. The rectangular-face transducer is embedded in an ellipsoidal housing. The transducer was designed and fabricated at the NIH High-Frequency Ultrasonic Transducer Technology Resource Center at USC. Seen from the side, the transducer has a $45^{\circ}$ angled press-focused face. Press-focusing was done using the method of Lockwood [11].

\subsection{Transducer Spectral Profile}

The transducer spectral profile is shown in Fig. 9. F1 and F2 are defined as lower and upper $-6 \mathrm{~dB}$ frequencies, at which the magnitude of the amplitude in the spectrum is $50 \%(-6 \mathrm{~dB})$ of the maximum. $\mathrm{F}_{\text {center }}$ was $20.7 \mathrm{MHz}$. F1 and F2 were, respectively, 15.5 and $25.8 \mathrm{MHz}$. For sound speed in water ( $c=1480 \mathrm{~m} / \mathrm{sec}$ ), the wavelength at $20 \mathrm{MHz}$ is 74 microns.

\subsection{VARS device assembly}

As shown in Figure 8, the mounting of the forward-imaging endoscope is achieved with two concentric separate stainless steel tubes, with inner tube \# 1 contained within outer tube \# 2. Tube \# 1 contains the transducer and is fixed in position. Rotating tube \# 2 has a $2 \mathrm{~mm}$ VARS surface strip micro-machined into the interior of its distal end. VARS tube \#2 rotates about transducer tube \#1.

The transducer $45^{\circ}$ beam is always directed toward a midpoint on the internal VARS surface strip. Between tube \# 1 and tube \# 2, there is a thin parylene coating (less than 3/1000 of an inch) applied along the length of the transducer tube and on the transducer aperture. Outer tube \# 2 is $8 \mathrm{~cm}$ long and has an outer wall thickness of $1.5 \mathrm{~mm}$. The motor shaft connects to tube \# 2 proximally at a point $2.5 \mathrm{~cm}$ inside the motor. This laser-welded connection connects the rotating $3 \mathrm{~mm}$ wide motor shaft to the shaft of tube \# 2 with its distal $5 \mathrm{~mm}$ wide VARS assembly.The rotational shaft of the VARS needle assembly is driven by a stepper motor (National Instruments, Austin, TX) at a rate of 1 revolution per 2 seconds. A thru-hole at the back of the transducer shaft is made for wire exiting. Rotation of the VARS tube \#2 about the fixed transducer tube results in a linear beam sweep.

\subsection{Experimental water bath and phantom wire targets}

An experimental water bath was filled with de-gassed and de-ionized water. Calibration of the transducer position was done before phantom target imaging. The VARS ultrasound transducer was vertically positioned and aligned so that the emitted US beams would shoot toward the center of each target - toward phantom wires initially. In the calibration, the $45^{\circ}$ face was used for initial referencing so that the US beam would be reflected in a horizontal $0^{\circ}$ direction. A vertical targeting surface (plastic plane) was positioned in front of the route of the beam. The position of the transducer was then finely adjusted by a 5-degree position stage. When the reflected signal achieved its maximum value, it meant the transducer was shooting directly at the center of the phantom wire. 
Phantom Target-A wire phantom target was positioned in front of the VARS endoscope, within the image view of the VARS as in Fig. 10 and 11. The wire phantom consisted of five $20 \mu \mathrm{m}$ diameter tungsten wires (California Fine Wire Co., Grover Beach, $\mathrm{CA})$, that were arranged diagonally with equal distance in axial $(1.55 \mathrm{~mm})$ and lateral $(0.65$ $\mathrm{mm})$ directions. Since the wavelength $(74 \mu \mathrm{m})$ of the $20 \mathrm{MHz}$ transducer is larger than the diameter of the tungsten wire, the wire could be treated as an ideal line target. The axial and lateral wire image width (measured at $-6 \mathrm{~dB}$, or where signal strength decreases to half of maximum), can be treated as the axial and lateral resolutions of the transducer.

\subsection{Signal generation and data processing}

A schematic of the experimental setup is presented in Fig. 12. A Panametrics PR5900 pulser/receiver (Olympus NDT, Inc., Kennewick, WA) was used to drive the transducer in transmitting and receiving ultrasound waves. Echo signals were digitized by a 12-bit data acquisition board (Gage Applied Technologies, Lockport, IL) with a sampling rate of 200 MHz. A function generator (Agilent Technologies, Inc., Santa Clara, CA), which was triggered by the stepper motor, provided synchronization signals to both the pulser/receiver and the data acquisition board. The scanning procedure was controlled by the LabVIEW program. 2000 A-lines were acquired during each VARS revolution. The raw data were saved and post processed for image display. In data post processing, each scan line represented the average of 5 adjacent A-lines in order to reduce noise level, and the images were morphologically converted to a $60^{\circ}$ view.

\subsection{Ex vivo cow eye study}

An ex vivo cow eyeball (Sierra Medical Inc., Whittier, CA) was freshly harvested, and immersed in de-gassed and de-ionized water. To visualize the anterior segment of the eye, a $20 \mathrm{MHz}$ VARS transducer was placed above sclera spur. The setup was similar to that used for the phantom wire experiment, except that chirp coded excitation was used [12-13]. The function generator (AFG3251, Tektronix, Beaverton, OR, USA) was combined with a power amplifier (75A250A, Amplifier Research, Souderton, PA), to serve as an arbitrary pulse generator. The excitation voltage was 75 volt (p-p) with a $50 \mathrm{ohm}$ load. The designed transducer's impedance was very close to $50 \mathrm{ohm}$, such that the applied voltage level was about 75 volts.

Chirp coded excitation method was employed to enhance echo signal to noise ratio (eSNR). The utilized chirp burst for a $20 \mathrm{MHz}$ VARS transducer swept frequency was from $10 \mathrm{MHz}$ to $30 \mathrm{MHz}$ during $2 \mu \mathrm{s}$. In data post processing, the echo was compressed by a matched filter (the matched filter is a time reversed transmitted chirp burst).

\subsection{Results}

Axial resolution (in depth) was determined by the duration of the ultrasound pulse within -6 $\mathrm{dB}$ of the spectral profile maxima. Mathematically it is given by $\mathrm{R}_{\mathrm{axial}}=\mathrm{c} /(2 \times \mathrm{BW})$, where $\mathrm{c}$ is the sound speed in the detected medium, and $\mathrm{BW}$ is the $-6 \mathrm{~dB}$ bandwidth of a transducer.

Lateral spatial resolution in elevation and azimuthal direction is defined as the beam profile in the lateral direction $-6 \mathrm{~dB}$ of the maxima, which can be expressed as $R_{\text {lateral }}=f_{\#} \times \lambda$, where $f_{\#}$ is the $f$-number of a transducer, defined as the ratio of focal distance to diameter of a transducer.

\subsection{VARS Phantom Wire Imaging Without Chirp Coded Excitation}

In an experimental water bath study of the central $\left(3^{\text {rd }}\right)$ phantom wire without chirp coded excitation (see Fig. 13), the axial resolution was $66 \mu \mathrm{m}$ at a depth of $10 \mathrm{~mm}$. Without chirp- 
coded excitation, the lateral resolution for the $20 \mathrm{MHz}$ transducer was $510 \mu \mathrm{m}$ at a depth of $10 \mathrm{~mm}$. Dynamic imaging was over a range of $40 \mathrm{~dB}$.

\subsection{VARS phantom wire imaging with chirp coded excitation}

In a separate experimental setup, a phantom-wire study (central $3^{\text {rd }}$ wire) was performed with chirp coded excitation (see Fig. 14). The axial resolution was $66 \mu \mathrm{m}$ at $10 \mathrm{~mm}$ depth, and the lateral resolution was $520 \mu \mathrm{m}$ at $10 \mathrm{~mm}$ depth over a $40 \mathrm{~dB}$ dynamic imaging range. Echo signal-to-noise ratio (eSNR) was increased by $8 \mathrm{~dB}$ using chirp coded excitation. This reference experimental setup was used immediately prior to the ex vivo eye study.

\subsection{VARS ex vivo cow eyeball study with chirp coded excitation}

A water bath VARS imaging study of an ex vivo excised cow eyeball was performed with chirp coded excitation. The position of the endoscope tip external to the eye is illustrated in Fig. 11. The resulting VARS image made possible the delineation and identification of the structures of the anterior segment (cornea, sclera, ciliary body, and iris), as shown in Fig. 15.

\subsection{Discussion}

\subsection{VARS feasibility and 'blind spot' elimination}

The present study demonstrates the feasibility of imaging with a needle-like device whereby beam-steering is achieved with a novel variable-angle reflecting surface (VARS). This firsteffort VARS device with its $5 \mathrm{~mm}$ diameter width is comparable in size to reported equally thin needle-like or catheter devices. Further reductions are possible at different levels in the width of component layers --motor shaft width, VARS thickness, transducer thickness--that could lead to a high-frequency overall device width of about 2.5 to $3 \mathrm{~mm}$. Such a device would likely be usable and welcome in the clinical performance of needle-based interventional procedures.

The VARS mechanism overcomes the 'forward blind spot' problem associated with beams emitted by angle-faced transducers. The VARS device achieves not only 'forward imaging', but achieves true 'straight-ahead' imaging. The micro-machining of the VARS surface is readily achievable with electric discharge machining (EDM).

\subsection{Resolution Matters and SNR benefits of chirp coded excitation system}

The reasons for using coded excitation are (1) to enhance the penetration depth and the eSNR of tissue images acquired with the VARS transducer, and (2) to limit the peak pressure amplitudes to non-injurious tissue levels. The tradeoff between image resolution and penetration depth can be lessened by the use of a long coded pulse and the use of a matched cross-correlation filter so as to compress the returning echoes in the time domain. Time compression improves the axial resolution and enhances SNR.

There are three major coded transmission techniques: Chirp coded excitation, Barker codes, and Golay codes [12]. In 'chirp' coded excitation, which is the method used in this study, there is a linear increase with time of the carrier frequency. This form of continuous phase modulation results in an autocorrelation function that features a single peak bordered on both sides by range side lobes. These side lobes generally are associated with image degradation. Appropriate weighting in the time or frequency domains can sometimes be helpful in reducing these chirp-associated side lobes. With chirp coded excitation, a single transmission at a time is made with the mechanical transducer.

In contrast to the chirp method, two transmissions are required for the Golay code, which relies upon pairs of complementary sequences, and which can be made to have no side lobes 
about the peak of the autocorrelation function. This method employs two codes of the same length $\mathrm{N}$ whose autocorrelation functions have side lobes equal in magnitude but opposite in sign. The side lobe summation results in an autocorrelation function with a peak of $2 \mathrm{~N}$ and zero side lobes[13]. The Barker code method, a discrete phase modulation technique, has an autocorrelation function peak of $\mathrm{N}$ and the side-lobe level falls between 1 and -1 .

Linear frequency modulation (FM) signals, such as chirp, are well suited for robust ultrasound imaging. Studies have indicated that the use of chirp-coded linear FM signals can result in an improvement in signal-to-noise ratio (SNR), with an expected SNR improvement of 12 to $18 \mathrm{~dB}$ for large imaging depths $(20 \mathrm{~cm})$ in attenuating media [14-16]. That is, the penetration depth is increased. For the shallow depth $(1-2 \mathrm{~cm})$ used in this study, eSNR was increased by $8 \mathrm{~dB}$ using chirp coded excitation. The higher SNR allows the use of higher frequencies that result in better image resolution.

For normal pulse excitation, the axial resolution is determined by the transducer's impulse response. For a coded system, the excitation pulse extends over a period much longer than the impulse response of the transducer. The received signal can then be correlated with a reference waveform to yield a compressed signal with an impulse response that approximates that of the normal pulsed system. For a chirp system, the acquired data are compressed by convoluting with a matched filter, namely, a time reversed transmitted waveform. The disadvantage of the correlator output is that it produces range side lobes that are not present in the simple pulse system. For example, such side lobes are absent in Fig. 13 (simple pulse), but are present in Fig. 14 (chirp coded system). For FM signals, the sharp edges of the rectangular spectrum generate the high, slowly decreasing side lobes of the sinc function. To achieve reduction in the size of the coded-system side lobes, various filtering and windowing techniques been developed, such as equalization filtering, mismatched filtering, or predistortion of the FM signal [15-16]. The design tradeoff in reducing the side lobes may be a widening of the main lobe and a decrease in SNR. For design guidelines on coded excitation, the reader is referred to the articles by Nowicki [12-13], O'Donnell [14], and Misaridis and Jensen [15-16].

Lateral resolution is affected by the width of the beam and by the depth of imaging. As the single-element transducer beam diverges further at greater depths, the lateral resolution decreases. Hence, the lateral resolution would be expected to be best at the shallowest depths. Generally the lateral resolution can be improved by increasing the frequency and by better focusing of the beam.

Although longitudinal needle axis imaging is the primary intended use of the device, it would be a simple matter for the needle operator to manually rotate the entire needle device through a desired angle to get a circumferential sense of the lateral surroundings, yet with longitudinal axial grade resolution. An alternative option, if available, would be to rotate only the internal transducer inside the VARS surface to get a 3-D sense of the surroundings, as illustrated in Fig. 6. This could be done without any manual rotation of the entire needle assembly device in the tissue, that is, the VARS surface would be left untouched in the same position while the internal transducer is rotated. These two maneuvers would likely be helpful in achieving high-grade partial imaging of lateral structures. We note that we are exploring ways to improve the axial and lateral resolution by further shaping of the VARS surface.

The temporal resolution, in terms of the frame rate, is described as frame rate $=$ (pulse repetition period) (number of scan lines per frame), or frame rate $\mathrm{F}=1 /$ (single frame scanning time). In this study, the frame rate $\mathrm{F}=1 \mathrm{fps}$, and the pulse repetition frequency $(\mathrm{PRF})=2 \mathrm{kHz}$. 
In the coded excitation image of Fig. 14, there also appears to be speckle in the image in the region closest to the transducer. Speckle results from a group of scatterers that are spaced much closer than the waveform resolution at the matched filter output and therefore cannot be resolved. Averaging cannot reduce the speckle signal as it does with noise [14]. We suspect the speckle is due to acoustic reverberation between the transducer and the VARS surface, but it could also be due to electrical noise, or due to an artifact of the filtering process.

There does not appear to be to be any evident major distortion of anatomic structures due to beam reflection from the rotating VARS surface per se with its built-in successive tilt angle changes.

\subsection{Imaging problems with US skin surface probes in regional anesthesia and pain blocks}

The recent advent of ultrasound (US) imaging in regional anesthesia practice, with its imaging of nerves and vessels and the needle insertion path, has resulted in higher block success rates, better onset times, reduced local anesthetic requirements, and a reduced incidence of complications [17-18]. Despite these advances in US usage for regional anesthesia, significant nerve imaging problems with these skin surface-placed transducer probes are encountered, as we describe below:

High-frequency US probes have high resolution but limited tissue imaging depth, and can be used only for superficial nerves, such as those seen in upper extremity plexus blocks. For deep locations, the associated attenuation and scattering problems would be markedly reduced if the US needle probe could be introduced through skin and its tip advanced to the structures of interest, and thus allow close-up visualization of neural structures such as fascicles [19].

In the treatment of cervical radicular pain, steroids and local anesthetics are injected in the vicinity of the spinal nerve root as it emerges from the intervertebral foramen. Major complications have been reported with needle injection into the cervical spinal neuroforamen, despite the use of fluoroscopic positioning: fatal spinal cord infarction, cortical blindness owing to vertebral artery puncture, quadriplegia, regional pain syndrome due to nerve root needle trauma, and fatal vertebral artery perforation [20-25]. Many cases have not been reported for medico-legal reasons. An ultrasound imaging technology is needed whereby real-time needle tip vision is achieved of critical neurovascular structures at the injection site. With such a capability, complications would be minimized, and the risk of procedure failure lessened.

In a recent study, an 18-gauge Tuohy epidural needle with an embedded $40 \mathrm{MHz}$ ultrasound angled-face non-VARS transducer was introduced via the lumbar paramedian approach, and A-mode imaging was used to identify and access the epidural space in a porcine model [26]. By inserting the high frequency needle ultrasound transducer into the hollow chamber of the epidural needle, the distance between the tip of the epidural needle, ligament flavum, and dura mater could be measured in real time by monitoring the reflected ultrasound signal in A-mode display. However, because imaging with this non-VARS needle exhibited a forward-imaging 'blind spot', the device had to be inserted at a non-direct angle, hence, the use of the paramedian approach. Had this device been equipped with a VARS capability, the needle could have been inserted directly into the midline intervertebral space instead of being inserted at an angle.

As described earlier, when complete VARS revolutions are integrated with successive incremental rotations of the single-element transducer, it should be possible to achieve 3-D VARS imaging with a single-element surface probe. This capability would make a VARS 
device especially useful in the performance of pain and regional anesthetic blocks, needle biopsies, drainage procedures, and procedures requiring entry into small orifices.

\subsection{Conclusions}

In this pilot study, we (1) proposed a novel built-in variable-angle reflecting surface (VARS) for use in 2-D and 3-D imaging, (2) fabricated a VARS prototype 2-D 'straight-ahead imaging' needle device with the use of available machining technology, (3) conducted experimental water bath experiments with phantom wire structures with and without chirpcoded excitation, and (4) implemented chirp-coded excitation in the high-resolution imaging of an ex vivo cow eye.

\section{Acknowledgments}

Support was made possible by National Institute of Health NINDS STTR Grant R41NS066542 to Sonoscrew, Inc. (Raphael PI) and P41-EB2182 to the University of Southern California (Shung PI).

Diagrams were made by Diane McIntee. The described VARS system was previously described by the first author in the U.S. patent application US 2011/0096628, which was published on April 28, 2011.

\section{References}

[1]. Curry, TS.; Dowdy, JE.; Murry, RC. Christensen's Introduction to the Physics of Diagnostic Radiology. third ed.. Lea and Febiger; Philadephia, PA: 1984. p. 391-393.

[2]. Shung, KK. Diagnostic ultrasound: imaging and blood flow measurements. CRC Press, Taylor and Francis Group; Boca Raton, Florida: 2006. p. 63-77.

[3]. Marhofer P, Greher M, Kapral S. Ultrasound guidance in regional anesthesia. Brit. J. of Anesth. 2005; 94:7-17.

[4]. Foster FS, Pavlin CJ, Lockwood GR, Ryan LK, Harasiewicz KA, Berube L, Rauth AM. Principles and applications of ultrasonic backscatter microscopy. IEEE Trans. Ultrason., Ferroelect., Freq. Contr. 1993; 40:608-616.

[5]. Knspik DA, Starkoski B, Pavlin CJ, Foster FS, A FS. 100-200 MHz ultrasound biomicroscope. IEEE Trans. Ultrason. Ferroelectr. Freq. Control. 2000; 47:1540-1549. [PubMed: 18238700]

[6]. Turnbull D, Starkowski BG, Harasiewicz KA, Semple JL, From L, Gupta AK, Sauder DN, Foster FS. A 40-100 MHz B-scan ultrasound backscatter microscope for skin imaging. Ultrasound Med. Biol. 1995; 21:79-88. [PubMed: 7754581]

[7]. Degertekin FL, Guldiken RO, Karaman M. Annular-ring CMUT arrays for forward-looking IVUS: transducer characterization and imaging. IEEE Trans. Ultrason., Ferroelect., Freq. Contrl. 2006; 53:474-82.

[8]. Nikoozadeh A, Wygant IO, Oraikan O, Ergun AS, Stephens AS, Thomenius KE, Dentinger AM, Wildes D, Akopyan G, Shivkumar K, Mahajan A, Sahn DJ, Khuri-Yakuv BT. Forward-looking intracardiac ultrasound imaging using a 1-D CMUT array integrated with custom front-end electronics. IEEE Trans. Ultrason., Ferroelect., Freq. Contrl. 2008; 55:2651-2660.

[9]. Cannata JM, Ritter TA, Chen W, Silverman RH, Shung KK. Design of efficient, broadband singleelement (20-80 MHz) ultrasound transducers for medical imaging applications. IEEE Trans. Ultrason., Ferroelect., Freq. Contr. 2003; 50:1548-1557.

[10]. Zhou Q, Wu D, Jing J, Hu C, Xu X, Williams J, Cannata JM, Lim L, Shung KK. Design and fabrication of PZN-7 \% PT single crystal high frequency angle needle ultrasound transducers. IEEE Trans. Ultrason., Ferroelect., Freq. Contrl. 2008; 55(6):1394-1399.

[11]. Lockwood GR, Turnbull DH, Foster FS. Fabrication of high frequency spherically shaped transducers. IEEE Trans. Ultrason., Ferroelect., Freq. Contr. 1994; 41:231-235.

[12]. Nowicki A, Klimonda Z, Lewandowski M, Litniewski J, Lewin PA, Trots I. Comparison of sound fields generated by different coded excitations-Experimental results. Ultrasonics. 2006; 44(1):121-129. [PubMed: 16313936] 
[13]. Nowicki A, Trots I, Lewin PA, Secommski W, Tymkiewicz R. Influence of the ultrasound transducer bandwidth on selection of the complementary Golay bit code length. Ultrasonics. 2007; 47:64-73. [PubMed: 17825338]

[14]. O'Donnell M. Coded excitation system for improving the penetration of real-time phased array imaging systems. IEEE Trans. Ultrason. Ferroelectr. Freq. Contr. 1992; 39:341-351.

[15]. Misaridis TX, Jensen JA. Use of modulated excitation signals in medical ultrasound. Part I: Basic concepts and expected benefits. IEEE Trans. Ultrason. Ferroelectr. Freq. Contr. 2005; 52:177191.

[16]. Misaridis TX, Gammelmark K, Jorgensen CH, Lindberg N, Thomsen AH, Pedersen MH, Jensen JA. Potential of coded excitation in medical ultrasound imaging. Ultrasonics. 2000; 38:183-9. [PubMed: 10829655]

[17]. Peter, Marhofer; Vincent, WS. Chan, Ultrasound-guided regional anesthesia: current concepts and future trends. Anesthesia Analgesia. 2007; 104:1265-1269. [PubMed: 17456684]

[18]. Hatfield A, Bodenham A. Ultrasound: an emerging role in anaesthesia and intensive care. Brit J of Anaesthesia. 1999:789-900.

[19]. Bigeleisen PE. Nerve puncture and apparent intraneural injection during ultrasound-guided axillary block does not invariably result in neurologic injury. Anesthesiology. 2006; 105:779_ 783. [PubMed: 17006077]

[20]. Baker R, Dreyfuss P, Mercer S, Bogduk N. Cervical transforaminal injection of corticosteroids into a radicular artery: a possible mechanism for spinal cord injury. Pain. 2003; 103:211-215. [PubMed: 12749976]

[21]. Brouwers P, Kottink E, Simon MAM, Prevo RL. A cervical anterior spinal artery syndrome after diagnostic blockade of the right C6-nerve roo. Pain. 2001; 91:397-399. [PubMed: 11275398]

[22]. Furman MB, O’Brien EM, Zgleszewski TM. Incidence of intravascular penetration in transforaminal lumbosacral epidural steroid injections. Spine. 2000; 25:2628-2632. [PubMed: 11034648]

[23]. Furman MB, Giovaniello MT, O'Brien EM. Incidence of intravascular penetration in transforaminal cervical epidural steroid injections. Spine. 2003; 28:21-25. [PubMed: 12544950]

[24]. Houten JK, Errico TJ. Paraplegia after lumbosacral nerve root block: Report of three cases. Spine Journal. 2002; 2:70-75. [PubMed: 14588291]

[25]. McMillan MR, Crumpton C. Cortical blindness and neurologic injury complicating cervical transforaminal injection for cervical radiculopathy. Anesthesiology. 2003; 99:509-511. [PubMed: 12883429]

[26]. HK Chiang Q, Zhou MS, Mandell MY, Tsou SP, Lin KK, Shung CK. Ting, Eyes in the needle: novel epidural needle with embedded high-frequency ultrasound transducer-epidural access in porcine model. Anesthesiology. 2011; 114:1320-4. [PubMed: 21519228] 


\section{HIGHLIGHTS}

Elimination of the 'blind spot' for angled face transducers

Design and fabrication of a rotating variable-angle reflecting surface (VARS) for 2-

D imaging

Use of VARS imaging in a prototype needle endoscope system 

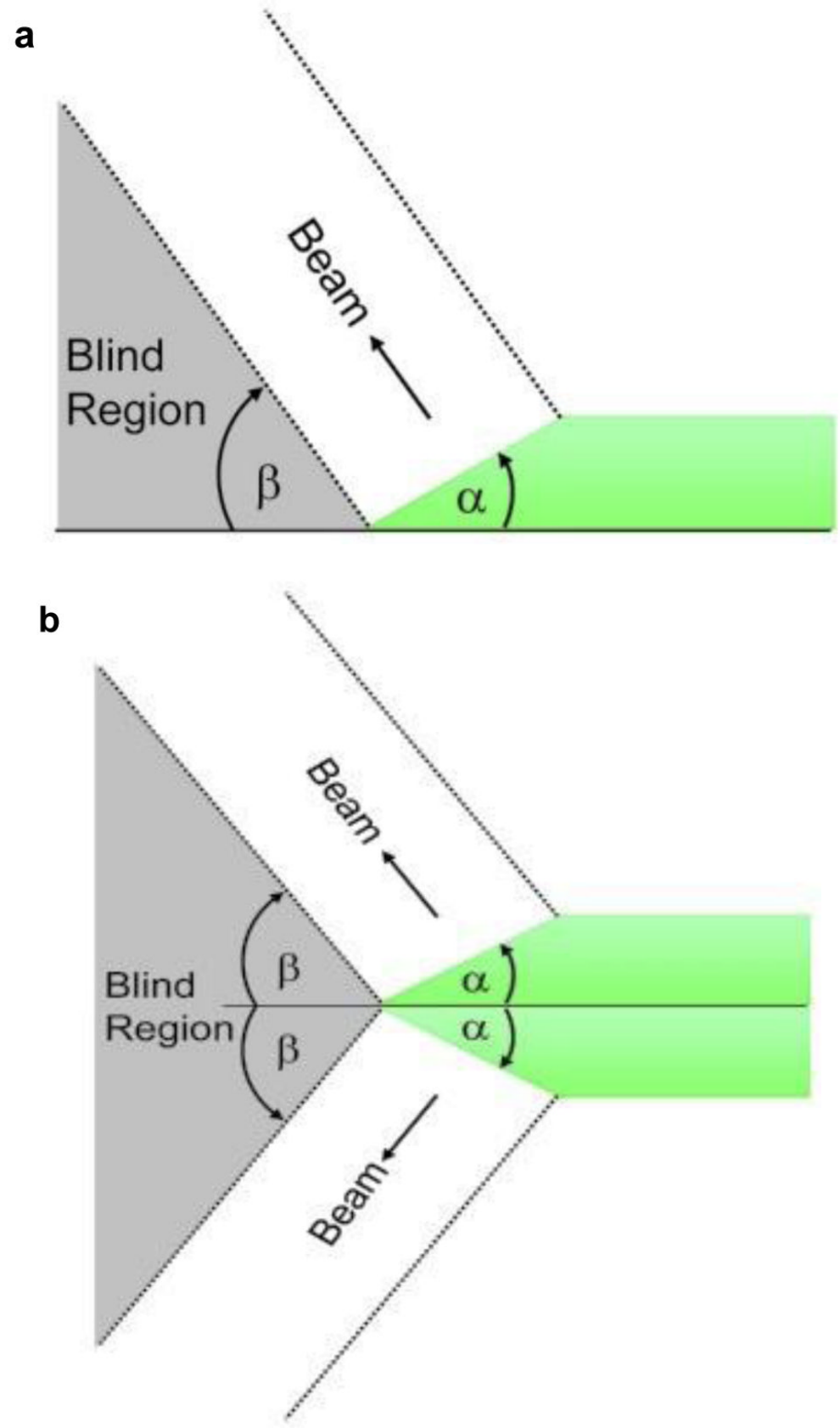

Fig. 1.

(a). A stationary angled-face transducer has a 2-D plane acoustic 'blind' region.

(b). Upon a complete rotation of the transducer, a 3-D cone-shaped 'blind' region is created. 


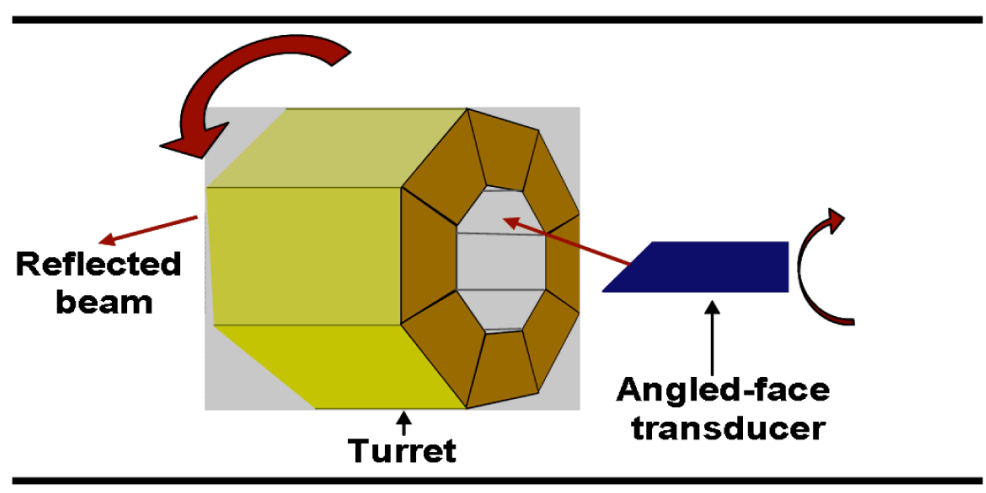

Fig. 2.

An angled-face transducer emits a beam that bounces off the inner, uppermost surface of a rotating hollow turret at the distal end of an endoscope. The beam is reflected forwards beyond the turret. The outer wall of the endoscope is indicated by two thick axial lines. 


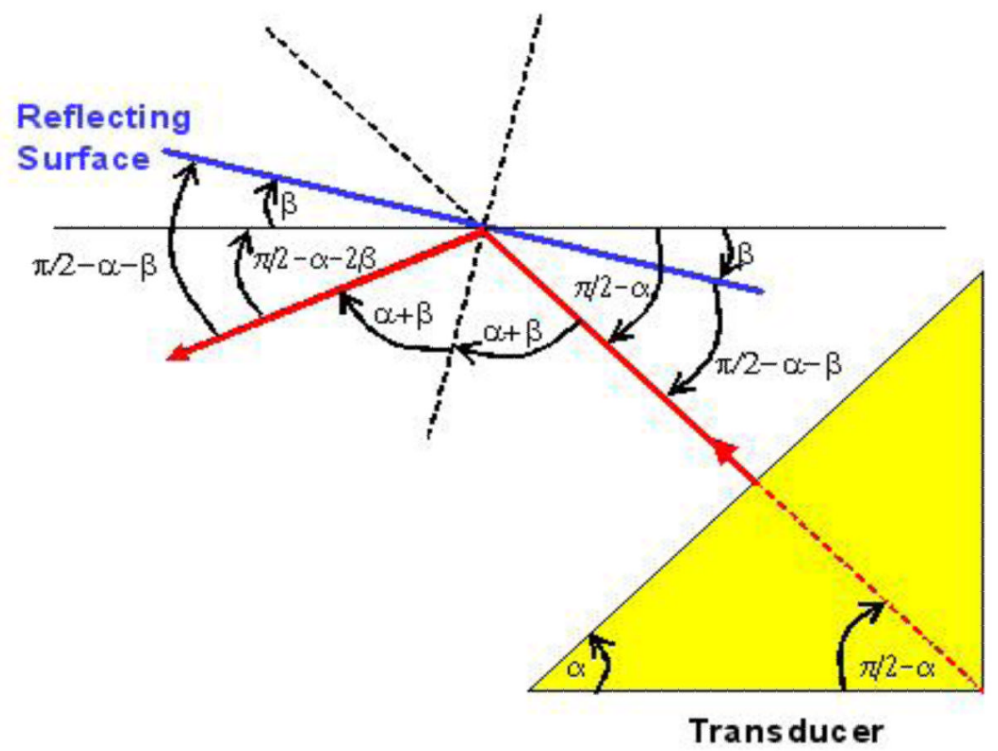

Fig. 3.

A beam is emitted at angle $a$ from the transducer face midpoint and is reflected at athe midpoint of a reflecting surface that has a variable tilt angle $\beta$. The reflected beam emerges with angle $(\pi / 2-a-2 \beta)$. 

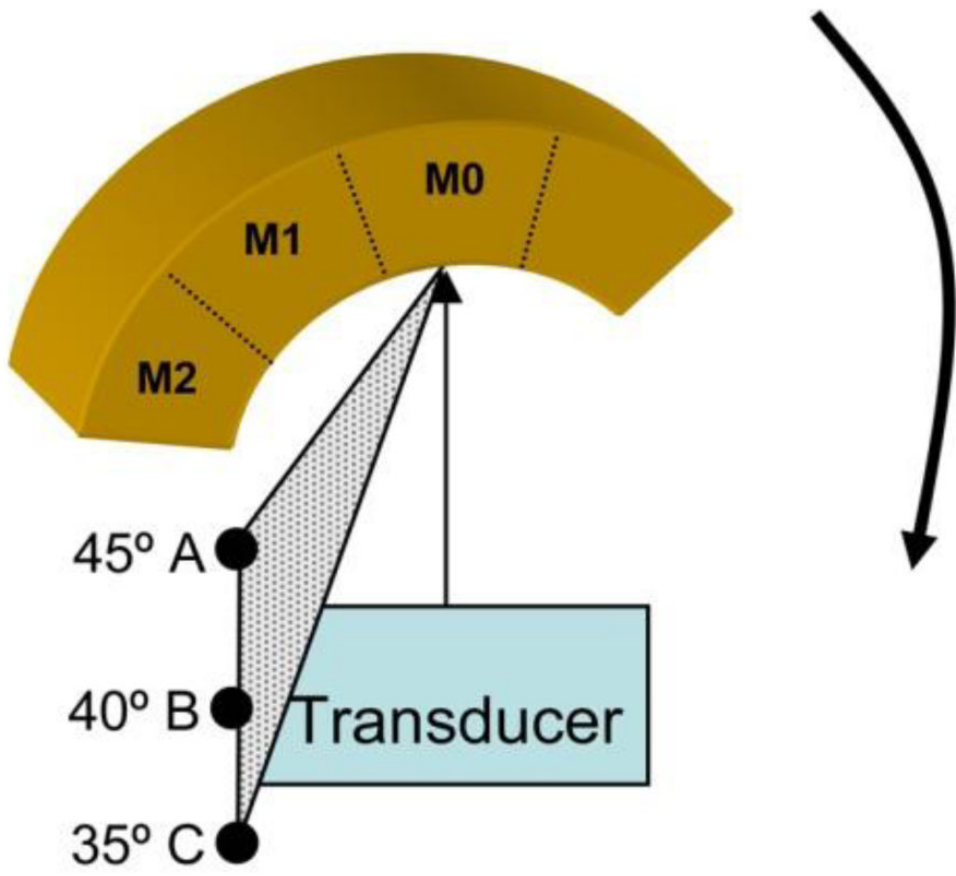

Fig. 4.

The transducer is located behind the turret. Mirrors M0, M1, M2 have different undersurface tilt angles $\left(0,2.5,5^{\circ}\right)$. For a stationary $45^{\circ}$ angled face transducer, rotation of each successive mirror into the uppermost position causes successive forward beam reflections of 45,50 , and $55^{\circ}$ with corresponding focal points $\mathrm{A}, \mathrm{B}, \mathrm{C}$ in a sector scan. 


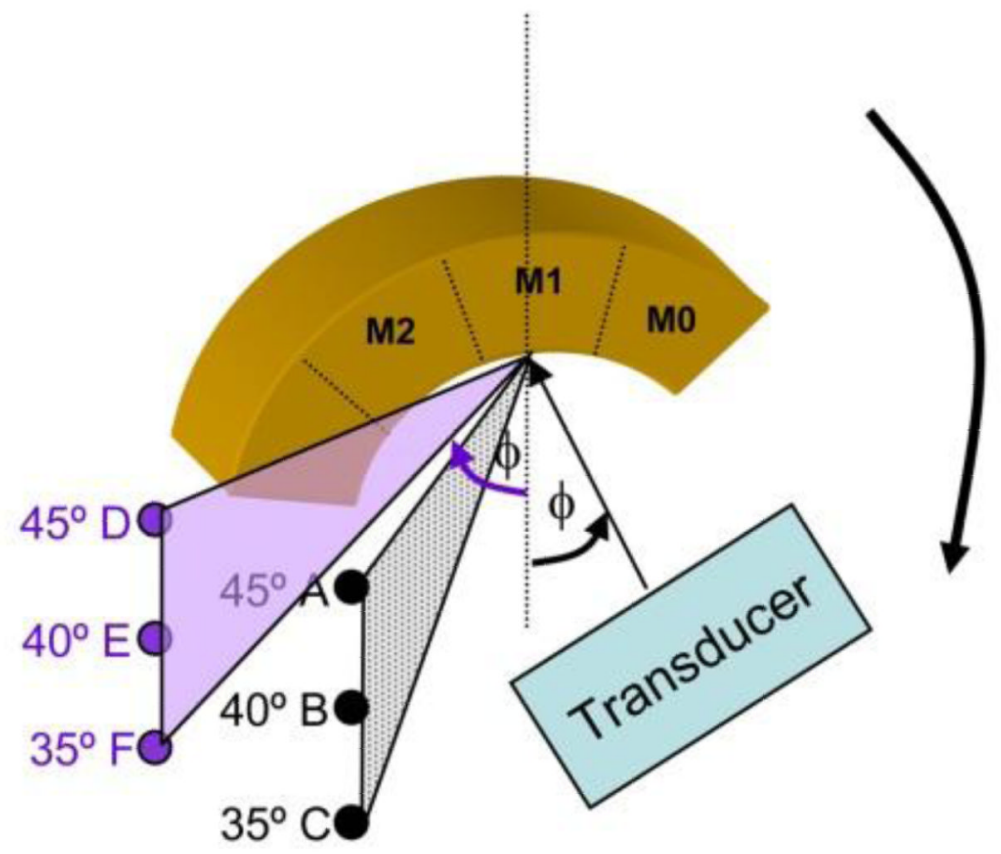

Fig. 5.

Rotation of the transducer by angle $\varphi$ creates focal points D, E, and F in a new 2-D scan. 


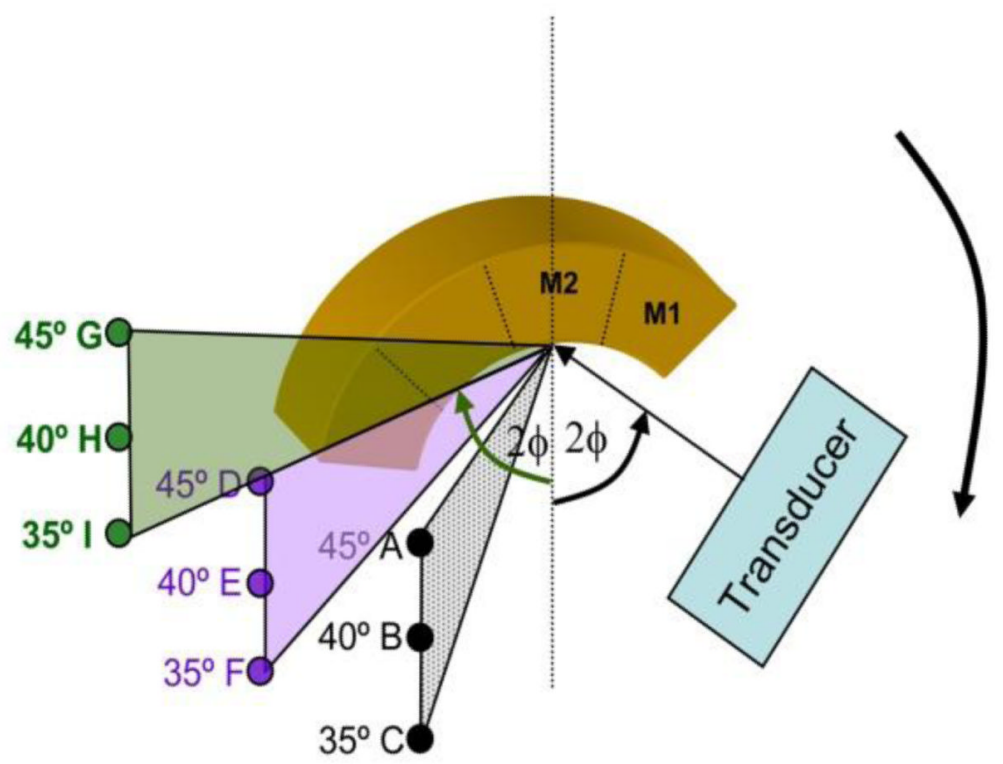

Fig. 6.

Rotation of the transducer by angle $2 \varphi$ creates focal points $G, H$, and I in another 2-D scan. 


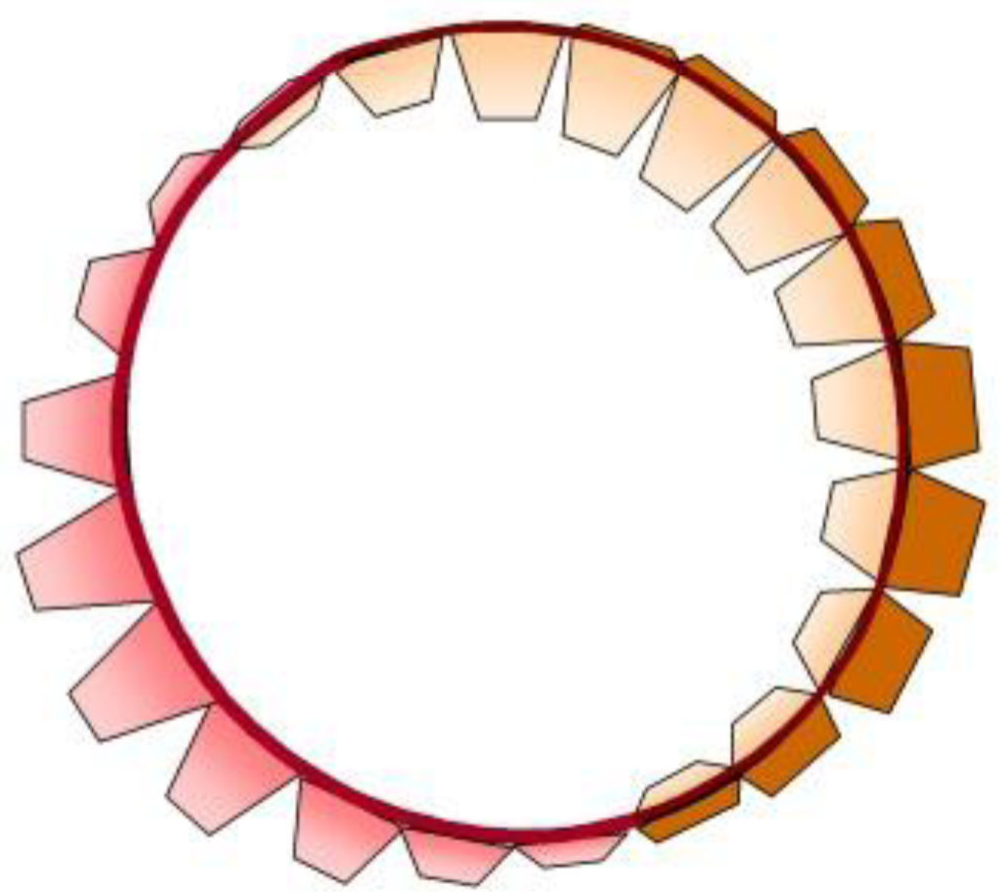

Fig. 7.

The Variable-Angle Reflecting Surface (VARS). For purposes of illustration, the VARS surface is discretized into 21 reflecting surfaces. The midpoints of all successive reflecting regions are constrained to the perimeter of a circular disc (the dark red line). All VARS reflecting surface midpoints are found on this circle. 


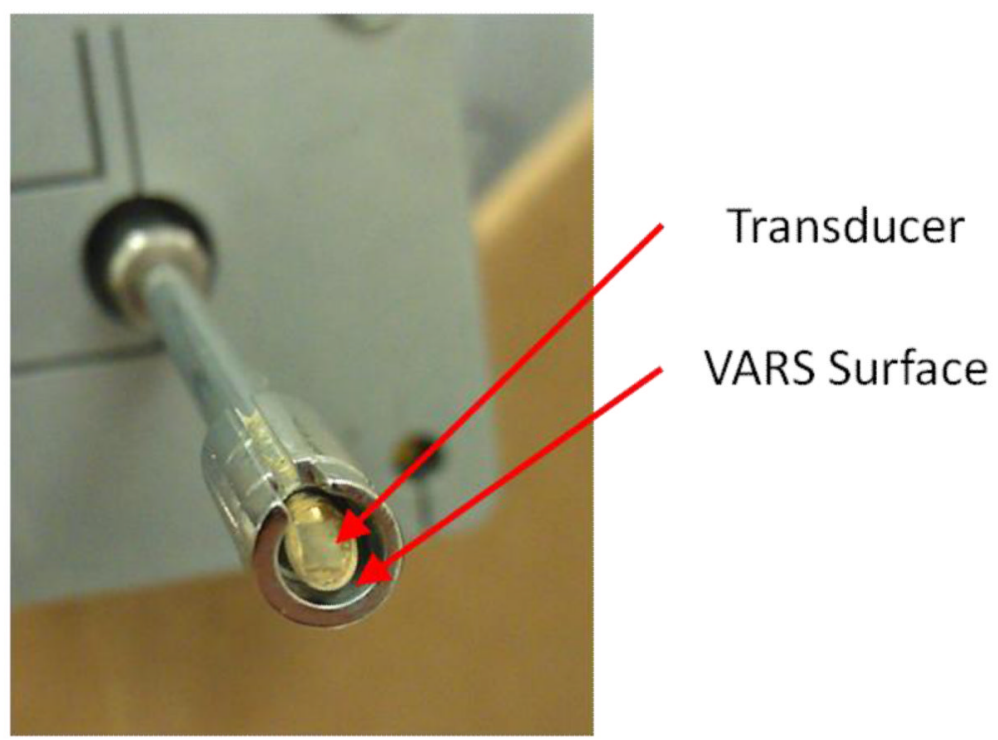

Fig. 8.

C.U. view of the internal VARS endoscope prototype. The VARS surface strip is micromachined into the inner surface of the distal end. The VARS strip rotates around the transducer. The PMN-PT transducer is angle-faced $\left(45^{\circ}\right)$, is of aperture size $1.15 \mathrm{~mm} \times 1.63$ $\mathrm{mm}$, and has a $7.5 \mathrm{~mm}$ focus. 


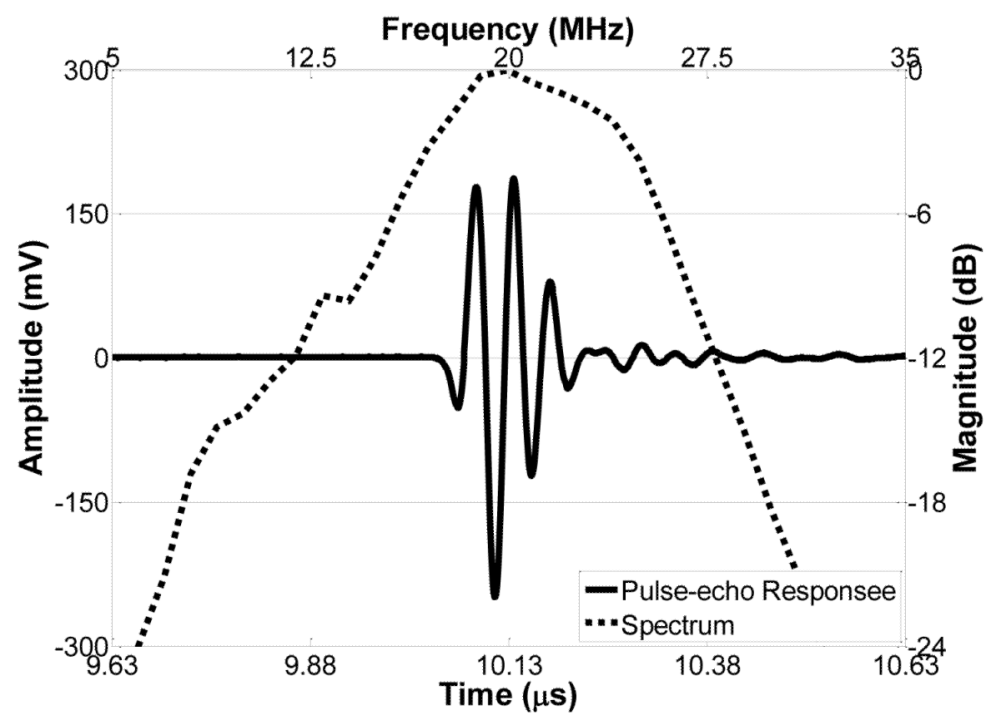

Fig. 9.

Spectral profile of the $20 \mathrm{MHz}$ PMN-PT transducer. 


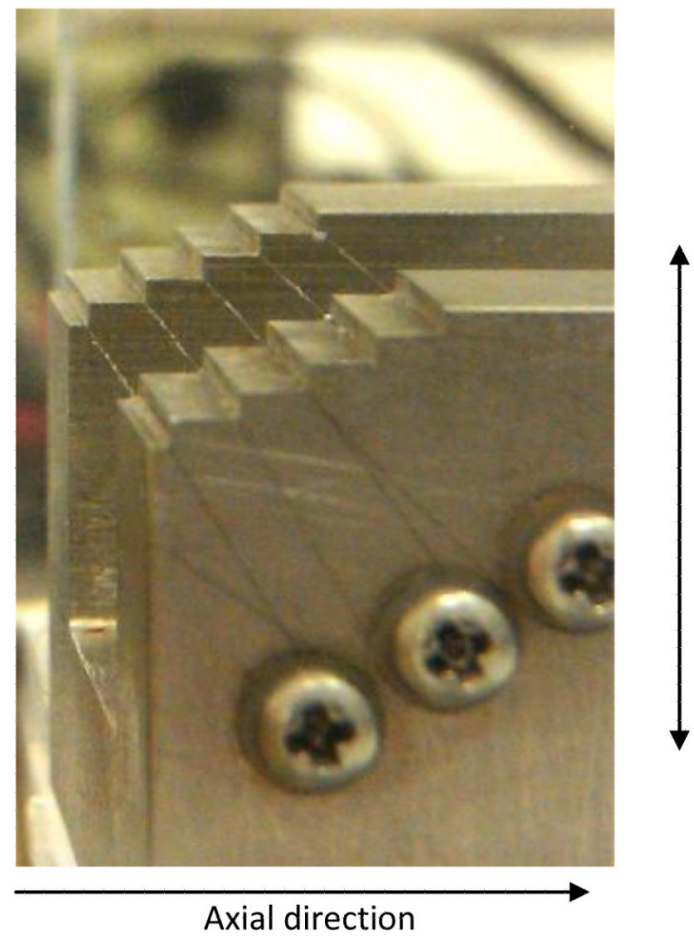

Lateral direction

Fig. 10.

Tiered phantom wires (20 $\mu \mathrm{m}$ diameter). 


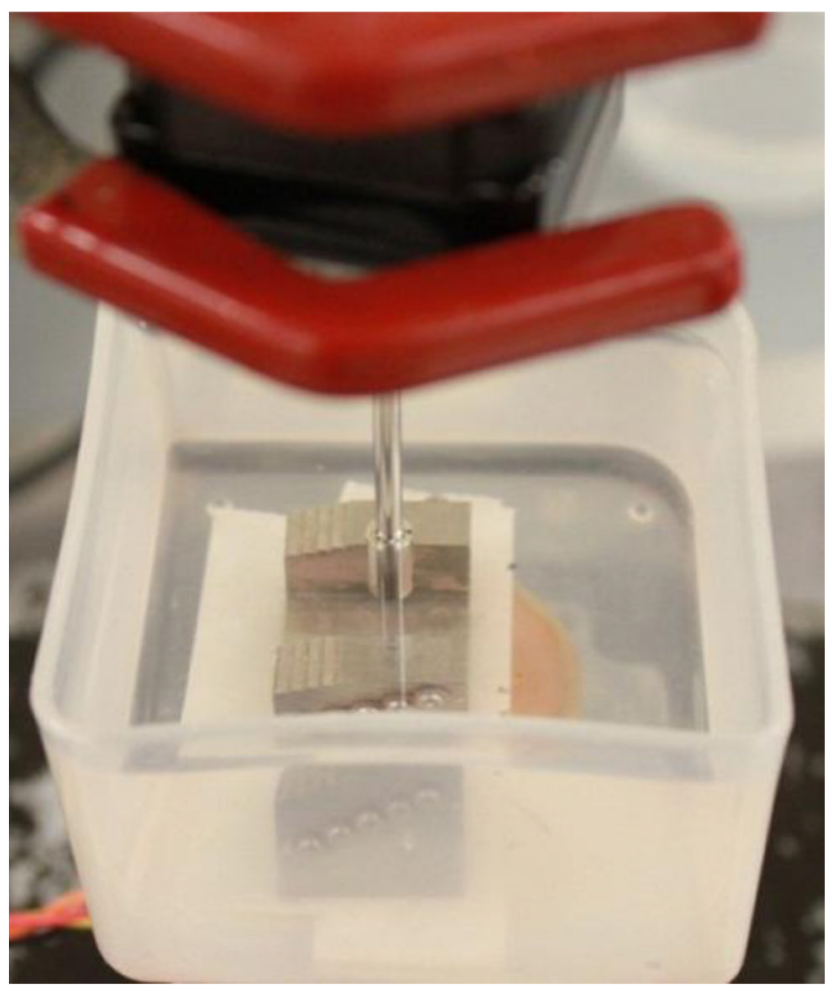

Fig. 11.

Inner VARS endoscope pointed toward the phantom wires. 


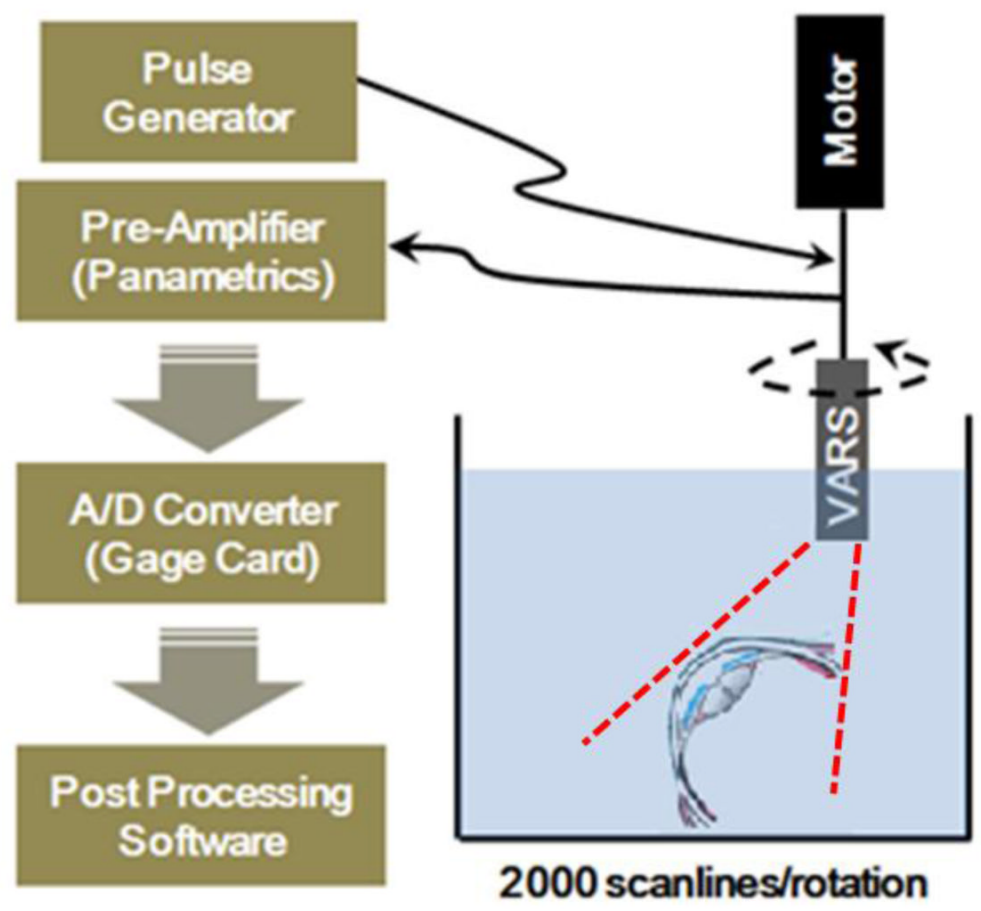

Fig. 12.

Experimental water bath setup for ex vivo cow eye study. A similar setup was used for the phantom wire target study. 


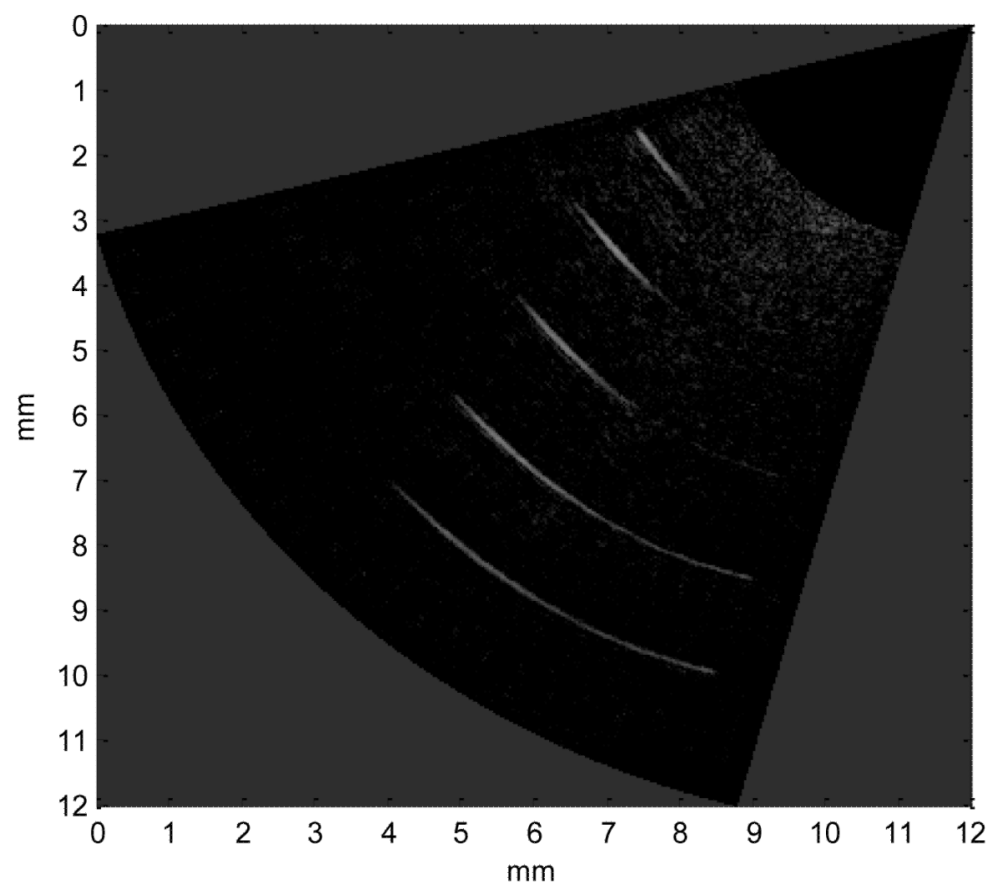

Fig. 13.

Phantom wire imaging ( $3^{\text {rd }}$ wire) with $20 \mathrm{MHz}$ VARS endoscope and $40 \mathrm{~dB}$ imaging range without chirp coded excitation. Axial resolution (third wire): $66 \mu \mathrm{m}$. Lateral resolution: 510 $\mu \mathrm{m}$. 


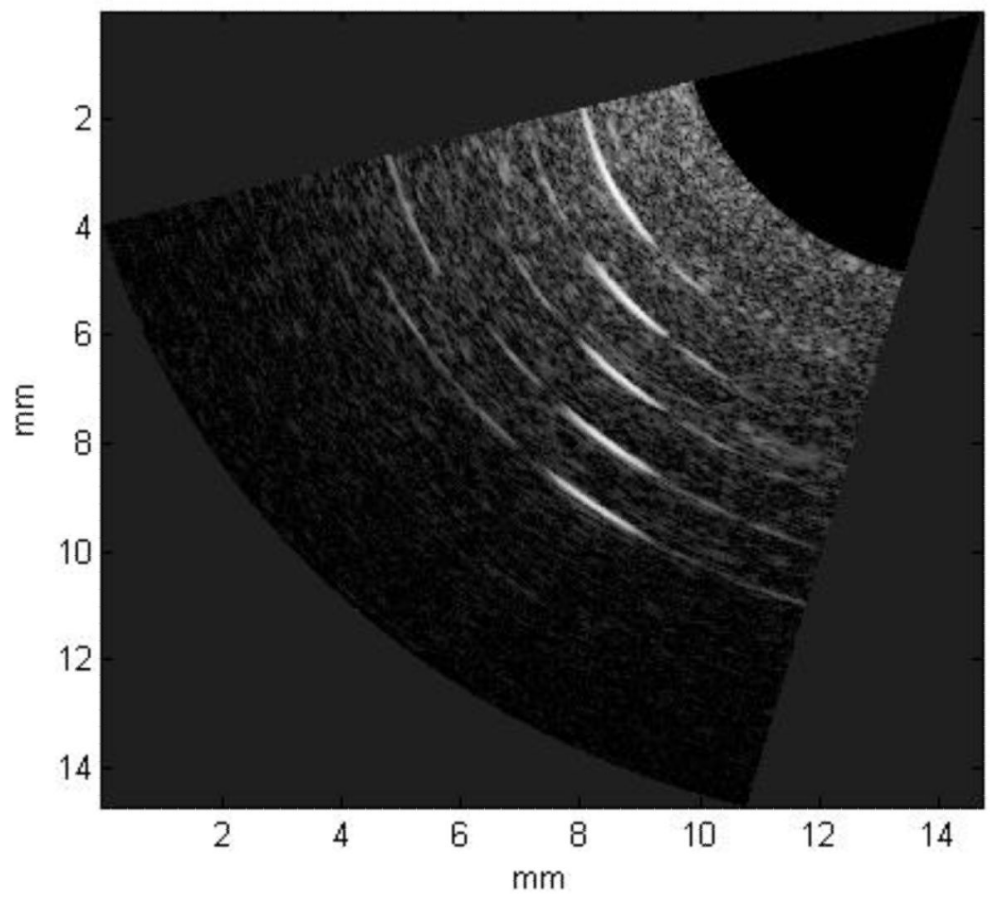

Fig. 14.

Phantom wire imaging ( $3^{\text {rd }}$ wire) with $20 \mathrm{MHz}$ VARS endoscope and chirp coded excitation over $40 \mathrm{~dB}$ imaging range. Axial resolution: $66 \mu \mathrm{m}$. Lateral resolution: $520 \mu \mathrm{m}$. 


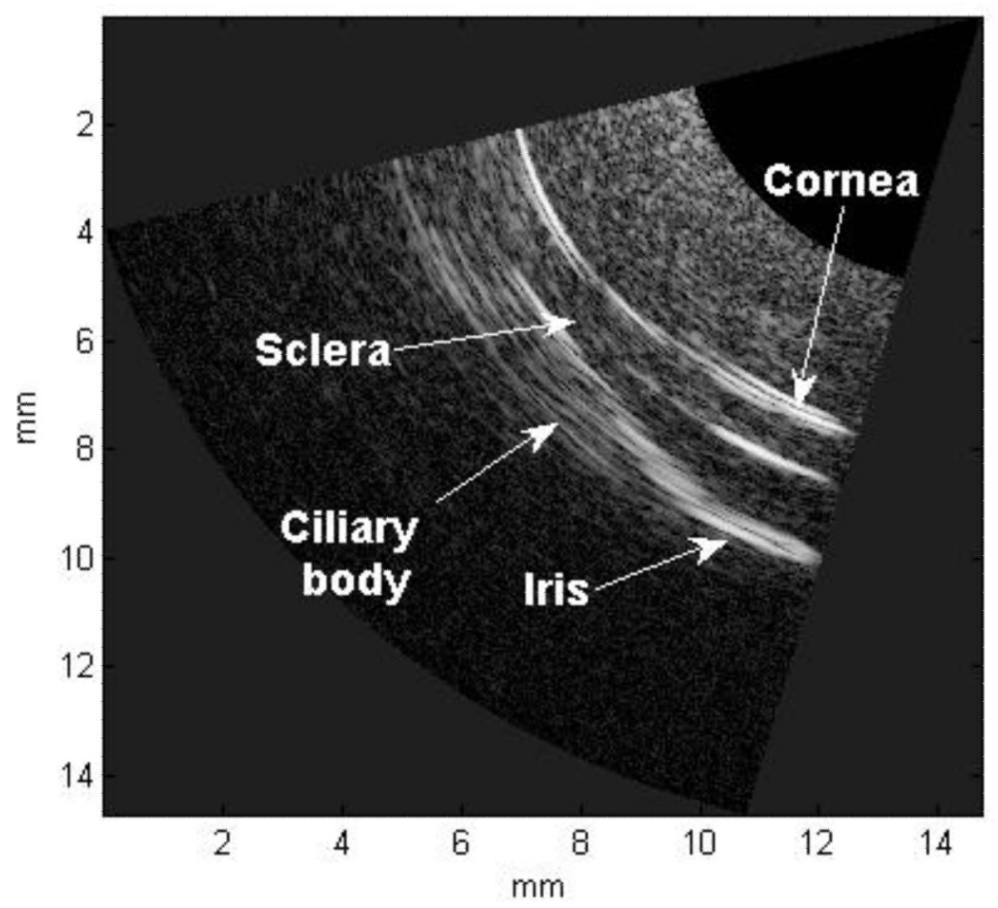

Fig. 15.

Cow eyeball image VARS image acquired with chirp coded excitation. The structures of the anterior segment (cornea, sclera, ciliary body, and iris) can be identified. 